\title{
Contribuciones teóricas sobre la metodología colaborativa para el tratamiento de los errores gramaticales de un texto académico en inglés en un contexto universitario
}

\author{
Theoretical Contributions on the Collaborative Methodology for the Grammar Error \\ Treatment of an English Academic Text in a University Context
}

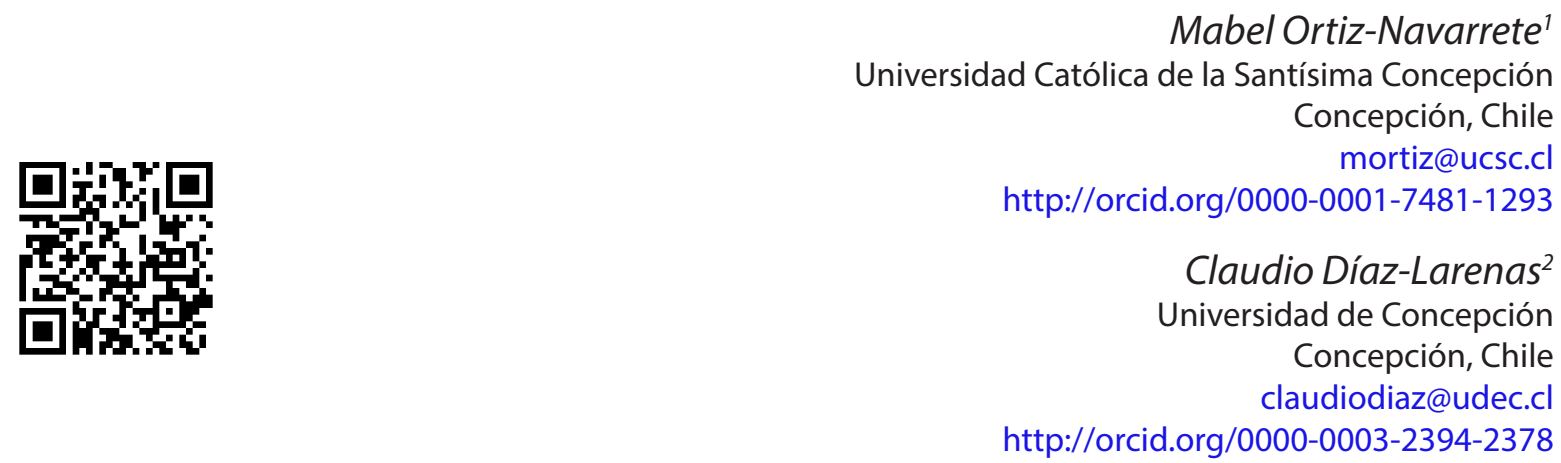

Recibido 17 de diciembre de 2015 • Corregido 16 de febrero de 2017 • Aceptado 19 de abril de 2017

\begin{abstract}
Resumen: El presente artículo tiene como objetivo destacar las características de la metodología colaborativa para el tratamiento de errores gramaticales en un texto académico en inglés. El artículo destaca los siguientes componentes: la interacción, el diálogo, la discusión, el andamiaje y la ZPD (zona desarrollo próximo) y la interdependencia positiva. Tales componentes propician, además, la participación activa de todos los miembros de un grupo en la corrección de errores gramaticales en inglés. Se enfatiza también en este ensayo que el trabajo colaborativo puede activar lo metalingüístico y potenciar el efecto del feedback correctivo que entrega cada docente a un grupo.
\end{abstract}

Palabras clave: Metodología colaborativa; texto académico; errores gramaticales.

${ }^{1}$ Profesora de inglés como lengua extranjera. Doctora en Lingüística y Magister en Tecnologías de la Información. Sus áreas de interés son el uso de las tecnologías en la enseñanza del inglés y la provisión de feedback correctivo en ambientes virtuales colaborativos. Posee artículos en ambas áreas. Actualmente es académica de la Universidad Católica de la Santísima Concepción, Concepción, Chile.

2 Profesor de inglés como lengua extranjera. Doctor en Educación y Magister en Lingüística. Sus áreas de interés son la formación de docentes y las creencias del profesorado en torno a la enseñanza del inglés en Chile. Posee artículos Scielo e ISI en el área. Ha liderado diversos proyectos de investigación relacionados con el área de la enseñanza del inglés en Chile. Actualmente es académico de la Universidad de Concepción, Concepción, Chile. 
doi: http://dx.doi.org/10.15359/ree.21-2.20

URL: http://www.una.ac.cr/educare

CORREO: educare@una.cr

\begin{abstract}
This article aims to highlight the characteristics of the collaborative methodology for error correction of $\mathrm{L} 2$ students'texts. The article points out the following components: interaction, dialogue, discussion, scaffolding, ZPD (Zone of proximal development), and positive interdependence; these components also favor the active participation of all members of a group during error correction. In addition, the paper emphasizes the way the collaborative work can activate the metalinguistic component, and can enhance the effect of corrective feedback provided by the teacher to a group.
\end{abstract}

Keywords: Collaborative methodology; academic text; grammar errors.

\title{
Introducción
}

La producción de textos académicos en inglés implica una actividad compleja que involucra la complementación de diversos factores, como la motivación, la creatividad, el desarrollo de destrezas lingüísticas y comunicativas, entre varios otros. Debido a esta convergencia, surge una problemática recurrente, esto es, los tipos de errores gramaticales que comete el estudiantado en la producción de textos académicos y la forma como estos deben ser abordados.

Si bien, la producción de un texto debe estar centrada principalmente en el discurso o mensaje que se desea transmitir, no se puede restar importancia a la precisión lingüística cuando el texto producido es de índole académico. Algunas investigaciones señalan que el profesorado universitario, en situaciones donde el idioma inglés no se encuentra en un contexto inmediato, como es el caso del contexto chileno, consideran que los errores gramaticales pueden influir negativamente en la comprensión de un texto (Ferris \& Roberts, 2001) y, por lo tanto, tienden a focalizarse con frecuencia en la corrección de estos aspectos. Las observaciones en el aula corroboran estas investigaciones, puesto que existe una tendencia generalizada del profesorado en la focalización de los errores, principalmente de tipo gramatical.

Por otra parte, abordar los errores dentro de una situación comunicativa evita enseñar la gramática de manera descontextualizada. Esto, dado que la necesidad comunicativa se aleja de lo que es la instrucción de la gramática y se centra en lo que se denomina 'la atención en la forma'. Es decir, si bien se mantiene la preocupación de los aspectos formales de la lengua, no se descuida el contenido del mensaje ni el contexto en el que se enmarca la interacción comunicativa. En este contexto, un error se caracteriza por ser sistemático y, por esta razón, requiere de un tratamiento específico por parte del personal docente, quien debe fomentar el avance de cada estudiante durante el proceso de adquisición de una L2. Al respecto, la manera de corregir los errores frecuentes que comete el estudiantado en la escritura es una de las tareas más complejas para el profesorado, más aún, cuando se trata de la corrección de distintos tipos de errores, como sucede comúnmente en la producción escrita.

Mucho se ha teorizado acerca de cómo debe abordarse el error durante el proceso de enseñanza y aprendizaje de una L2. Entre estas teorías se encuentra el dilucidar cuál debe ser la manera docente de actuar frente a un error del estudiantado. Ello no solamente en el contexto 
de las estrategias de feedback, sino que también en la toma de decisiones con respecto a qué metodología puede ser la más apropiada para abordarlos. Lo anterior supone un gran reto para el profesorado, puesto que, además surge la disyuntiva sobre si la corrección es solamente un papel que le compete al profesorado o el estudiantado también puede desempeñar un rol importante en esta fase de la producción de un texto.

Desde esta perspectiva, el presente ensayo describe el aprendizaje colaborativo como una metodología que posee elementos que pueden facilitar el tratamiento de errores gramaticales, a saber: el diálogo que involucra la interacción y discusión de ideas, el andamiaje y la ZPD. Se destaca también que la metodología colaborativa puede activar el metalenguaje y potenciar el efecto del feedback correctivo en la corrección de errores.

Este artículo se inserta en el contexto de los proyectos: Fondecyt N. ${ }^{0}$ 11150273: Uso de estrategias de feedback correctivo focalizado en un entorno virtual colaborativo. Impacto y plan de acción (Ortiz, 2015) y DIN 01/2013: El uso de estrategias de feedback correctivo y su efecto en la adquisición de aspectos gramaticales durante la escritura colaborativa de ensayos en un entorno virtual wiki (Ortiz, 2013).

\section{Desarrollo}

\section{El diálogo en el tratamiento de errores}

La metodología colaborativa considera el diálogo como una herramienta adecuada para el logro de las metas. Un aspecto importante por considerar es que durante el diálogo el estudiantado se ayuda entre sí en la solución de los problemas que surgen a propósito de los aspectos de forma. La cantidad de estudiantes propicia el desarrollo de las tareas grupales. En el contexto de una tarea de escritura, el número de participantes puede ser aún más significativo, dado lo complejo que puede resultar el desarrollo de este tipo de tareas en idioma inglés y más aún con un fin académico. Una tarea de escritura colaborativa puede ser enriquecedora para el estudiantado en la medida en que promueve el desarrollo de diversas habilidades. Así, por ejemplo, se desarrollan las habilidades sociales, puesto que durante el proceso de escritura deben interactuar y llegar a acuerdos con el fin de elaborar un texto único. El diálogo en una tarea de escritura colaborativa tiene relevancia no solo en la elaboración y organización de ideas, sino también para resolver problemas lingüísticos y, por ende, puede llegar a ser un elemento indispensable tanto en la comunicación de un mensaje como en la adquisición de estructuras gramaticales. Lo anterior es corroborado por Swain (2000), quien señala que el expresar una idea o un mensaje verbalmente y el dialogar constituyen dos acciones favorables para la adquisición del lenguaje, especialmente en la adquisición de aspectos gramaticales. 
doi: http://dx.doi.org/10.15359/ree.21-2.20

URL: http://www.una.ac.cr/educare

CORREO: educare@una.cr

La oportunidad de dialogar en los procesos de escritura tiene varias ventajas en relación con la escritura individual. En efecto, en la escritura colaborativa se puede argumentar, compartir conocimientos y resolver dudas, entre otras acciones. Tales espacios son vitales para potenciar tanto el contenido como los aspectos formales de un texto escrito. Algunos estudios como los de Kowal y Swain (1994), Swain (2000), Swain y Lapkin (2001) apoyan el uso de tareas colaborativas como una actividad que facilita la identificación de errores lingüísticos durante la fase dialógica. Esta instancia permite que el estudiantado hable sobre la lengua y discuta sobre los errores lingüísticos. En concreto, la función social de la producción escrita y la activación de los procesos de reflexión y negociación del significado pueden facilitar el proceso de corrección de errores. Al respecto, Stein, Bernas y Calicchia (1997) señalan que durante una tarea de escritura colaborativa, el estudiantado se hace más consciente de su proceso de aprendizaje y "use each other as a source of knowledge" [se usan unos a otros como fuente de conocimiento] (p. 233) durante la discusión y negociación de ideas, lo que puede tener una incidencia positiva en el tratamiento de los errores en un texto escrito. durante la discusión y negociación de ideas, lo que puede tener una incidencia positiva en el tratamiento de los errores en un texto escrito.

Durante la fase de revisión, el diálogo y la interacción grupal son relevantes. En esta fase el estudiantado debe releer un texto y puede ser una etapa crucial para la identificación de errores. Considerando las características del trabajo colaborativo, el grupo en conjunto puede revisar todo el escrito o cada integrante lo puede hacer por separado. En esta etapa se pueden asignar roles en que cada miembro asume una responsabilidad en la corrección. El aspecto gramatical, que puede requerir un nivel de revisión de mayor dificultad, puede ser compartido por todo el grupo. En suma, a través de la interacción, quienes aprenden deberían desarrollar la capacidad de resolver los problemas que surgen durante la redacción de un texto en colaboración. Esta oportunidad dialógica con los pares permite lo que no es posible en la escritura individual, esto es: argumentar, compartir conocimiento y resolver dudas, entre otras acciones.

\section{El andamiaje y la ZPD en la corrección de errores}

Un concepto clave de la metodología colaborativa es el 'andamiaje'. Este es utilizado para ilustrar el proceso en el que el estudiantado es apoyado en su aprendizaje, ya sea a través de un profesor o profesora, un compañero o compañera o durante el trabajo en equipo. En el ámbito de la corrección de errores, algunas investigaciones llevadas a cabo han demostrado cómo el andamiaje basado en la colaboración y la interacción puede contribuir al reconocimiento de errores y a la autocorrección (Lai \& Zhao, 2006; Smith, 2008; Warschauer, 2010). En consecuencia, la responsabilidad de apoyar al estudiantado en la construcción del conocimiento relacionado con la corrección de errores no recae solamente en el profesorado. Por otra parte, a través de observaciones en el aula, se puede señalar que en reiteradas ocasiones el estudiantado comprende mejor la explicación de su par que la que puede entregar su docente. Esto, en el 
contexto de errores, puede ser muy relevante, puesto que estudiantes con un alto grado de timidez no siempre se sienten con la confianza suficiente para consultar a su profesor o profesora sobre los errores que comete y, por lo tanto, tiende a recurrir a sus pares. No se puede, entonces, desconocer que los factores emocionales inciden en la adquisición de una segunda lengua.

Otro concepto relevante es la zona de desarrollo próximo (ZPD) que Vygotsky (1999) utiliza para referirse a lo que cada estudiante puede lograr cognitivamente con la ayuda de pares más capaces. Al respecto, la metodología colaborativa sugiere, en la medida de lo posible, grupos heterogéneos. Por ende, grupos compuestos por miembros con diferentes habilidades permitirán que personas novatas sean guiadas por expertas 0 , en otras palabras, que estudiantes más débiles puedan fortalecerse con la ayuda de estudiantes con mayores habilidades o conocimientos. En el contexto de la corrección de errores, esta conformación de grupo puede facilitar la disipación de dudas en torno a los errores gramaticales, que son muy comunes en estudiantes de inglés como lengua extranjera. Esto, principalmente, por las diferencias evidentes entre la estructura del idioma inglés y del español. Los errores frecuentes encontrados en los textos escritos corresponden a preposiciones, uso de auxiliares, tiempos verbales, entre otros. En este escenario, es común que el estudiantado avance en su competencia comunicativa; pero no así en su competencia gramatical. Por lo tanto, la búsqueda o utilización de nuevas metodologías para abordar los errores, como puede ser la metodología colaborativa, es relevante en la enseñanza y aprendizaje del inglés.

\section{La interdependencia positiva y la corrección de errores}

La interdependencia positiva definida como "la relación que se establece entre los integrantes de un grupo para compartir procesos y resultados" (Ferreiro, 2004, p. 163) conlleva a que el estudiantado dependa entre sí para el logro de las metas. En el contexto de la corrección de errores esta interdependencia puede facilitar un mayor involucramiento de los miembros de un grupo para atender y corregir un error, puesto que cada integrante tiene una responsabilidad individual que debe favorecer el logro del objetivo que tienen en común. En una tarea individual, cada estudiante no siente la presión del trabajo en equipo y, por lo tanto, puede perfectamente no atender un error, como comúnmente ocurre en una tarea de escritura.

Si bien, el estudiantado generalmente trabaja en grupo, la totalidad no comparte las mismas responsabilidades, lo que se traduce en que siempre hay alguien del grupo que trabaja más que el resto. Este es el concepto erróneo de trabajo colaborativo que comúnmente conoce el estudiantado y, por lo tanto, no lo percibe como una metodología que se destaca por la existencia de una interdependencia positiva y de una participación equitativa. 
doi: http://dx.doi.org/10.15359/ree.21-2.20

URL: http://www.una.ac.cr/educare

CORREO: educare@una.cr

\section{El metalenguaje}

Un aspecto relevante a destacar de la metodología colaborativa es su cualidad para activar lo metalingüístico. Cuando se activa lo metalingüístico, la atención se dirige al lenguaje mismo. Esta acción conlleva una reflexión más profunda y más consciente sobre lo que se está aprendiendo. En la escritura colaborativa de un texto, dicha información metalingüística es una instancia en que la lengua pasa a ser objeto de estudio. Dada esta característica, las actividades en pequeños grupos permiten que estudiantes hablen por más tiempo acerca de la lengua. Camps, Guasch, Milian y Ribas (2000) señalan que la interacción permite la activación de la función metalingüística y, por lo tanto, tiene un rol primordial en la adquisición de elementos lingüísticos. En otras palabras, la interacción es un elemento relevante en el aprendizaje de distintos aspectos de la lengua, en este caso la gramática.

Por lo tanto, el trabajo en grupo es una instancia que promueve la focalización en el lenguaje cuando se lleva a cabo una tarea. Esta atención en los aspectos formales durante el diálogo es denominada por algunas investigaciones como 'episodios relacionados con la lengua'. De este modo, se genera una instancia propicia para discutir en torno a los errores y disipar dudas, principalmente si existe una meta en común, que en este caso es el texto escrito. Al respecto, esta oportunidad de centrarse en la lengua permitiría que el estudiantado tome más conciencia sobre sus procesos mentales que finalmente lo llevarán a poner en práctica no solo su conocimiento pragmático de la lengua, sino que también su conocimiento declarativo o explícito, esto es, el conocimiento que comprende componentes como la gramática, el léxico y la fonología. Del mismo modo, al existir una conciencia explícita de la estructura de la lengua, el estudiantado puede detectar aspectos que pueden pasar inadvertidos, como ocurre normalmente en una tarea individual, donde si no cuenta con el feedback de su docente no siempre advierte sus errores y es muy probable que cometa una mayor cantidad de errores que cuando lleva a cabo una tarea colaborativa donde un par puede advertir dicho error. Esto puede ser más frecuente aún en el estudiantado que posee una baja competencia gramatical. En reiteradas ocasiones, no advierte algún tipo de error, lo que es producto de su limitado conocimiento lingüístico. Por lo tanto, para ese tipo de estudiante es primordial una metodología de trabajo en que la retroalimentación provenga solamente del personal docente.

\section{El feedback correctivo y la metodología colaborativa}

Finalmente, la metodología colaborativa posee características que pueden potenciar el feedback correctivo entregado por el profesorado. Los estudios en el contexto del aprendizaje colaborativo evidencian que la metodología colaborativa presenta características que pueden potenciar el feedback entregado por el profesorado, puesto que ella se encontraría inserta dentro de un contexto social, en el que los grupos de estudiantes trabajan colaborativamente para resolver problemas lingüísticos (Nassaji y Swain, 2000). En otras palabras, el efecto del feedback

6

Mabel Ortiz-Navarrete y Claudio Díaz-Larenas

Los artículos de la Revista Electrónica Educare del Centro de Investigación y Docencia en Educación de la Universidad Nacional, Costa Rica, se comparten bajo términos de la Licencia Creative Commons: Reconocimiento, No Comercial, Sin 0bra Derivada 3.0 Costa Rica. Las autorizaciones adicionales a las aquí delimitadas se pueden obtener en el correo: educare@una.cr 
puede ser mucho más significativo, dado que el estudiantado puede potenciar sus habilidades lingüísticas, ayudarse entre sí y tomar decisiones en conjunto, principalmente cuando quienes son más débiles en el uso del idioma inglés no comprenden los comentarios del profesorado. De esta manera, la colaboración en torno a la corrección de errores puede ayudar a resolver muchos de los problemas de fosilización lingüística, fenómeno frecuente en el estudiantado que aprende una lengua con estructuras muy diferentes a la L1. Por lo tanto, la metodología colaborativa en función de la corrección de errores podría ayudar a transitar más rápidamente por los diferentes estados de aprendizaje de la lengua extranjera (García-Heras, 2004).

Estudios relacionados con la provisión de feedback a nivel individual son numerosos, sin embargo, la provisión de feedback a nivel grupal ha sido escasamente explorada (Bunderson y Sutcliffe, 2003; Kozlowski y Klein, 2000; London y Sessa, 2006). Esto puede deberse a alguna dificultad para visualizar el aprendizaje desde un punto de vista social, menos aún, en el ámbito del tratamiento de errores. A pesar de esta evidencia, el feedback en la corrección grupal de errores, si bien puede tener algunos inconvenientes como cualquiera estrategia de enseñanza, también tiene ventajas. London y Sessa (2006) afirman que un inconveniente que se puede presentar en el procesamiento del feedback es que los integrantes de un grupo reaccionen individualmente o que tomen decisiones sin una discusión previa. Sin embargo, la base del aprendizaje colaborativo es la responsabilidad compartida, por lo tanto, este inconveniente no debería ocurrir si la tarea colaborativa se planifica y diseña de manera adecuada. Un feedback dirigido a un grupo puede ser recibido con mayor receptividad y atención. Ello ocurre puesto que el feedback escrito por parte del profesorado no necesariamente significa que el estudiantado atenderá los comentarios ni que realizará los cambios respectivos, más aún, cuando la cantidad de comentarios a veces excede la capacidad estudiantik para atender a todos ellos. Bartram y Walton (1994, p. 43) declaran que "las ventajas de promover la corrección por parte de los compañeros son diversas. El estudiantado se siente más involucrado, aprende a ser más independiente, y se reduce con ello el tiempo de intervención del profesor". Se produce también lo que se denomina en la metodología colaborativa como interdependencia positiva.

Ahora bien, puede ser difícil lograr un nivel de compromiso entre la totalidad de integrantes de un grupo, pero la metodología colaborativa es una forma de incentivar una mayor participación. Dicho nivel de compromiso en la corrección de errores, después del feedback entregado por el personal docente, es muy relevante. Ello, dado que el estudiantado, al ayudarse entre sí para resolver sus dudas, prescinden del rol facilitador del profesorado y recurren a sus pares para la resolución de problemas. La provisión de feedback grupal obliga al estudiantado a reunirse con los miembros del grupo con el fin de cumplir con los objetivos planteados y tomar decisiones. 
doi: http://dx.doi.org/10.15359/ree.21-2.20

URL: http://www.una.ac.cr/educare

CORREO: educare@una.cr

La Figura 1 sintetiza lo planteado en este ensayo.

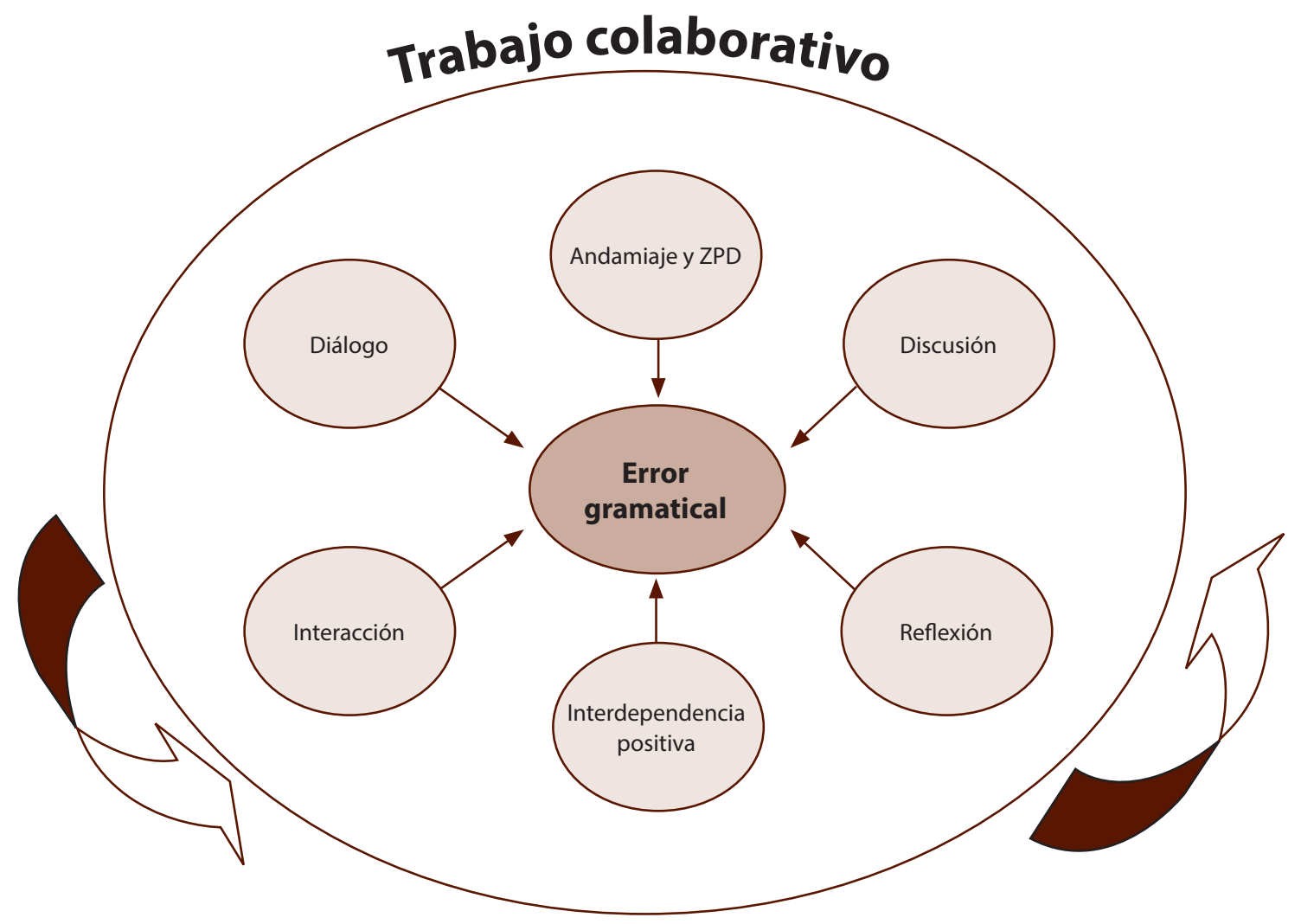

Figura 1: Tratamiento del error en un contexto colaborativo.

Nota: Elaboración propia.

\section{Algunas consideraciones finales}

Comprender el aprendizaje como un fenómeno social, en el sentido que lo plantea Vygotsky (1999), puede no ser compartido por el profesorado ni por el estudiantado. Sin embargo, es necesario abrirse a nuevos escenarios de aprendizaje y propiciar más instancias de colaboración en el aula para el desarrollo de la escritura y la corrección de errores. En este contexto, (1) el estudiantado debe ser entrenado en el uso de metodologías vinculadas al trabajo colaborativo, las que van más allá de un simple trabajo en grupo. Asimismo, (2) debe ser familiarizado con aprendizajes de tipo social y dialógico, con el fin de que (3) asuma un rol más activo dentro de sus procesos de aprendizaje. En suma, trabajando conjuntamente, los tres elementos pueden mejorar los aprendizajes, sobre todo si son utilizados de forma sistemática y frecuente. 
La escritura colaborativa fomenta el uso de la lengua en contextos significativos. En el proceso de adquisición de una L2, además del feedback que entrega el personal docente y la participación activa del estudiante, se hace necesaria la búsqueda de distintas estrategias para desarrollar y potenciar la metacognición y la autorregulación en el estudiantado. Una de ellas es, justamente, la escritura colaborativa, la que fomenta el uso de la lengua en contextos más significativos y, a la vez, potencia el feedback que entrega el profesorado. El tratamiento de los errores gramaticales dentro de un contexto colaborativo podría ayudar a prevenir muchos de los errores lingüísticos que comete el estudiantado en sus escritos, puesto que otorga una instancia en que se combinan distintos elementos, tales como la discusión, la interacción, la disipación de dudas, la retroalimentación con el fin de resolver problemas de estancamiento de la lengua, especialmente en grupos que poseen una baja competencia gramatical.

El rol docente en el tratamiento de errores durante el aprendizaje de una L2 es fundamental. Se hace imprescindible que transmita, en su discurso, los procesos internos que ocurren durante el aprendizaje de una lengua, tales como los fenómenos de fosilización y de interlengua. Ahora bien, el tipo de feedback que entregue estará determinado por el nivel de proficiencia del estudiantado, puesto que para un estudiantado no nativo que posee un nivel de conocimiento básico de la L2, resultaría incomprensible la información metalingüística.

En Chile, las condiciones contextuales en la sala de clases no benefician el tratamiento de errores por parte del profesorado. En efecto, la cantidad de estudiantes en la sala de clases y el número de horas en el aula no permite al profesorado dedicar tiempo suficiente para corregir los errores de un texto escrito y, por lo tanto, el avance en el aprendizaje se ve estancado. La escritura colaborativa de un texto permitirá que el proceso de enseñanza sea compartido entre el profesorado y el estudiantado, asumiendo este último grupo un rol más activo.

Finalmente, se puede concluir que el personal docente debería promover en el aula el aprendizaje colaborativo, especialmente, a través de la agrupación heterogénea de los grupos con el fin de conformar colectivos con distintas habilidades para abordar la corrección de errores. De esta manera, por una parte, se instan los procesos de interacción y de ZPD y, por otra, se potencia el feedback que entrega el profesorado, así como también, la acción de andamiaje.

\section{Referencias}

Bartram, M., \& Walton, R. (1994). Correction: A positive approach to language mistakes. England: Language Teaching Publications.

Bunderson, J. S., \& Sutcliffe, K. M. (2003). Management team learning orientation and business unit performance. Journal of Applied Psychology, 88(3), 552-560. doi: https://doi. org/10.1037/0021-9010.88.3.552 
doi: http://dx.doi.org/10.15359/ree.21-2.20

URL: http://www.una.ac.cr/educare

CORREO: educare@una.cr

Camps, A., Guasch, O., Milian, M., \& Ribas, T. (2000). Metalinguistic activity: The link between writing and learning to write. En A. Camps, \& M. Milan (Eds.), Metalinguistic activity in learning to write (pp. 103-124). Amsterdam: Amsterdam University Press.

Ferreiro, E. (2004). Alfabetización. Teoría y práctica (6ª ed.). México: Siglo XXI.

Ferris, D., \& Roberts, B. (2001). Error feedback in L2 writing classes: How explicit does it need to be? Journal of second language writing, 10(3), 161-184. Doi: https://doi.org/10.1016/ S1060-3743(01)00039-X

García-Heras, A. (2004). Lingüística y enseñanza: El tratamiento de errores en la enseñanza de inglés como lengua extranjera. Docencia e Investigación, 29(14), 49-70.

Kowal, M., \& Swain, M. (1994). Using collaborative language production tasks to promote students' language awareness 1. Language Awareness, 3(2), 73-93. doi: https://doi.org/10. $\underline{1080 / 09658416.1994 .9959845}$

Kozlowski, S., \& Klein, K. J. (2000). A multilevel approach to theory and research in organizations: Contextual, temporal, and emergent processes. In S.W.J. Kozlowski \&, K. J., Klein (Eds.), Multilevel theory, research, and methods in organizations: Foundations, extensions, and new directions (pp. 3-90). San Francisco: Jossey-Bass.

Lai, C., \& Zhao, Y. (2006). Noticing and text-based chat. Language Learning \& Technology, 10(3), 102-120. Recuperado de http://lt.msu.edu/vol10num3/pdf/laizhao.pdf

London, M., \& Sessa, V. I. (2006). Group feedback for continuous learning. Human Resource Development Review, 5(3), 303-329. doi: https://doi.org/10.1177/1534484306290226

Nassaji, H., \& Swain, M. (2000). A Vygotskian perspective on corrective feedback in L2: The effect of random versus negotiated help on the learning of English articles. Language Awareness, 9(1), 34-51. doi: https://doi.org/10.1080/09658410008667135

Ortiz, M. (2013). El uso de estrategias de feedback correctivo y su efecto en la adquisición de aspectos gramaticales durante la escritura colaborativa de ensayos en un entorno virtual wiki (Proyecto de Investigación DIN 01/2013). Concepción, Chile: Universidad Católica de la Santísima Concepción.

Ortiz, M. (2015). Uso de estrategias de feedback correctivo focalizado en un entorno virtual colaborativo. Impacto y plan de acción (Proyecto de Investigación Fondecyt № 11150273). Concepción, Chile: Universidad Católica de la Santísima Concepción.

Smith, B. (2008). External links valid at time of publication methodological hurdles in capturing CMC data: The case of the missing self-repair. Language Learning \& Technology, 12(1), 85-103. 
Stein, N. L., Bernas, R. S., \& Calicchia, D. (1997). Conflict talk: Understanding and resolving arguments. In T. Givón (Ed.), Conversation: Cognitive, communicative and social perspectives (Typological studies in language, vol. 34, pp. 233-268). Amsterdam: John Benjamins.

Swain, M. (2000). The output hypothesis and beyond:Mediating acquisition through collaborative dialogue. In J. P. Lantolf (Ed.), Sociocultural theory and second language learning (pp. 97114). Amsterdam: John Benjamin.

Swain, M., \& Lapkin, S. (2001). Focus on form through collaborative dialogue: Exploring task effects. In C. N. Candlin (Ed.), Researching pedagogic tasks. Second language learning, teaching and testing (99-118). London: Taylor y Francis.

Vygotsky, L. S. (1999). Tool and sign in the development of the child. In R. W. Kieber (Ed.), The collected works of L. S. Vygotsky (Vol. 6: Scientific Legacy, pp. 3-68). New York: Kluwer Academic/Plenum.

Warschauer, M. (2010). Invited commentary: New tools for teaching writing. Language Learning \& Technology, 14(1), 3-8. Recuperado de http://ltt.msu.edu/vol14num1/commentary.pdf 